\title{
Approximation with modified Phillips operators
}

\author{
Danyal Soybaş \\ Department of Mathematics Education, Faculty of Education, Erciyes University, Kayseri 38039, Turkey. \\ Communicated by D. Baleanu
}

\begin{abstract}
In the present paper, we study modified Phillips operators in simultaneous approximation. The operators discussed here are important as they have link with the well-known Szász operators. We estimate some direct results for the operators. (c) 2017 All rights reserved.
\end{abstract}

Keywords: Szász operators, Phillips operators, simultaneous approximation, modulus of continuity, moment generating function, asymptotic formula, error estimation.

2010 MSC: 41A25, 41A30.

\section{Introduction}

In order to generalize the well-known Bernstein polynomials to the positive real axis Szász [16] introduced the following operators

$$
S_{\alpha}(f, x)=\sum_{k=0}^{\infty} e^{-\alpha x} \frac{(\alpha x)^{k}}{k !} f\left(\frac{k}{\alpha}\right), x \in[0, \infty) .
$$

These operators are linear positive operators and play an important role in the theory of approximation. Recently, Gupta [6] discussed some approximation properties of the operators $S_{\alpha}(f, x)$. Four years later Phillips [15] proposed a generalization of the Szász operator in the following form:

$$
L_{\alpha}(f, x)=e^{-\alpha x} f(0)+\alpha \sum_{k=1}^{\infty} s_{\alpha, k}(x) \int_{0}^{\infty} s_{\alpha, k-1}(t) f(t) d t, x \in[0, \infty),
$$

where $s_{\alpha, k}(x)=e^{-\alpha x} \frac{(\alpha x)^{k}}{k !}$. Later Mazhar and Totik [12], Finta and Gupta [1], Gupta and Srivastava [8], Govil et al. [4], Heilmann and Tachev [10], Gupta [5], Tachev [17], etc. discussed several approximation properties of the operators $L_{\alpha}(f, x)$. Recently, in order to generalize the Phillips operators, based on the parameter $\rho>0$, Păltănea in [13] proposed the following operators

$$
L_{\alpha}^{\rho}(f, x)=\int_{0}^{\infty} k_{\alpha}^{\rho}(x, t) f(t) d t=e^{-\alpha x} f(0)+\sum_{k=1}^{\infty} s_{\alpha, k}(x) \int_{0}^{\infty} \theta_{\alpha, k}^{\rho}(t) f(t) d t, x \in[0, \infty),
$$

Email address: danyal@erciyes.edu.tr (Danyal Soybaş)

doi:10.22436/jnsa.010.11.18 
where with $\delta(t)$ being Dirac delta function the kernel is given as

$$
k_{\alpha}^{\rho}(x, t)=\sum_{k=1}^{\infty} s_{\alpha, k}(x) \theta_{\alpha, k}^{\rho}(t)+\delta(t) e^{-\alpha x}
$$

and the basis functions are defined as

$$
s_{\alpha, k}(x)=e^{-\alpha x} \frac{(\alpha x)^{k}}{k !}, \theta_{\alpha, k}^{\rho}(t)=\frac{\alpha \rho}{\Gamma(k \rho)} e^{-\alpha \rho t}(\alpha \rho t)^{k \rho-1},
$$

and $f: I \rightarrow \mathbb{R}$, where $I=[0, \infty)$ is integrable function for which the above series and integrals are convergent.

Let us denote

$$
W=\left\{f: I \rightarrow \mathbb{R}, f \text { is integrable and there exist } M>0, q \geqslant 0:|f(t)| \leqslant M e^{q t}, t \geqslant 0\right\} .
$$

It was observed by Păltănea [13] that for any $\alpha>0, f \in W$ and any $b>0$, there is $\rho_{0}>0$ such that $L_{\alpha}^{\rho}(f, x)$ exists for all $\rho>\rho_{0}$ and

$$
\lim _{\rho \rightarrow \infty} L_{\alpha}^{\rho}(f, x)=S_{\alpha}(f, x)
$$

uniformly for $x \in[0, b]$. The operators (1.1) preserve constant as well as linear functions. As a special case if $\rho=1$ these operators reduce to the well-known Phillips operators. Also, it was proved in [13] that the operators $L_{\alpha}^{\rho}(f, x)$ preserve convexity of higher order and have the property of simultaneous approximation on compact sets. Very recently Gal and Gupta [2] established some results in complex domain of these operators.

The present article is the extension of the previous work of [13]. Here the aim of the present paper is to study some direct results in simultaneous approximation, which include asymptotic formula and an error estimate in terms of the modulus of continuity.

\section{Basic results}

In the sequel, we need the following basic lemmas:

Lemma 2.1 ([1]). For $m \in \mathbb{N}^{0}$ if we define

$$
\mu_{\alpha, m}(x)=\sum_{k=0}^{\infty} s_{\alpha, k}(x)\left(\frac{k}{\alpha}-x\right)^{m},
$$

then there holds the recurrence relation

$$
\mu_{\alpha, m+1}(x)=x\left[\mu_{\alpha, m}^{\prime}(x)+m \mu_{\alpha, m-1}(x)\right] .
$$

Further $\mu_{\alpha, \mathrm{m}}(\mathrm{x})=\mathrm{O}\left(\alpha^{-[(\mathrm{m}+1) / 2]}\right)$, where $[\mathrm{s}]$ denotes the integral part of $\mathrm{s}$.

Lemma 2.2 ([14]). For fixed $\alpha>0$ and $\rho>0$, if we denote $T_{\alpha, m}^{\rho}(x)=L_{\alpha}^{\rho}\left(e_{m}, x\right), e_{m}(t)=t^{m}, m=1,2, \cdots$ for $\mathrm{m} \in \mathbb{N}_{0}$ and $x \geqslant 0$, then

$$
\mathrm{T}_{\alpha, \mathrm{m}}^{\rho}(x)=\left(x+\frac{\mathrm{m}-1}{\alpha \rho}\right) \mathrm{T}_{\alpha, \mathrm{m}-1}^{\rho}(\mathrm{x})+\frac{x}{\alpha}\left[\mathrm{T}_{\alpha, \mathrm{m}-1}^{\rho}(\mathrm{x})\right]^{\prime}
$$

Further,

$$
\mathrm{T}_{\alpha, \mathrm{m}}^{\rho}(x)=x^{\mathrm{m}}+\frac{(\rho+1)}{2 \alpha \rho} \cdot \mathrm{m}(\mathrm{m}-1) x^{\mathrm{m}-1}+\frac{(\rho+1)}{24(\alpha \rho)^{2}} \cdot \mathrm{m}(\mathrm{m}-1)(\mathrm{m}-2)[(3 \mathrm{~m}-5) \rho+3 \mathrm{~m}-1] x^{\mathrm{m}-2}+\cdots .
$$

Remark 2.3. We may note here that the moment generating function of the operators (1.1) is given by $\mathrm{L}_{\alpha}^{\rho}\left(e^{\mathrm{At}}, x\right)$ and we can find the moments in alternate form as 


$$
\begin{aligned}
\mathrm{L}_{\alpha}^{\rho}\left(e^{A t}, x\right)= & \exp \left(\frac{\alpha x\left[(\alpha \rho)^{\rho}-(\alpha \rho-A)^{\rho}\right]}{(\alpha \rho \rho-A)^{\rho}}\right) \\
= & 1+x A+\frac{1}{2}\left(\frac{(1+\rho) x}{\alpha \rho}+x^{2}\right) A^{2}+\frac{\left(2 x+3 \rho x+\rho^{2} x+3 \alpha \rho x^{2}+3 \alpha \rho^{2} x^{2}+\alpha^{2} \rho^{2} x^{3}\right) A^{3}}{6 \alpha^{2} \rho^{2}} \\
& +\frac{\left(6 x+11 \rho x+6 \rho^{2} x+\rho^{3} x+11 \alpha \rho x^{2}+18 \alpha \rho^{2} x^{2}+7 \alpha \rho^{3} x^{2}+6 \alpha^{2} \rho^{2} x^{3}+6 \alpha^{2} \rho^{3} x^{3}+\alpha^{3} \rho^{3} x^{4}\right) A^{4}}{24 \alpha^{3} \rho^{3}} \\
& +O\left(A^{5}\right) .
\end{aligned}
$$

From the above expansion, we observe that the m-th order moment is given by

$$
L_{\alpha}^{\rho}\left(e_{m}, x\right)=\left[\frac{\partial^{m}}{\partial A^{m}} L_{\alpha}^{\rho}\left(e^{A t}, x\right)\right]_{A=0} .
$$

Lemma 2.4. For fixed $\alpha>0$ and $\rho>0$, if we denote $\mathrm{U}_{\alpha, \mathrm{m}}^{\rho}(\mathrm{x})=\mathrm{L}_{\alpha}^{\rho}\left((\mathrm{t}-\mathrm{x})^{\mathrm{m}}, \mathrm{x}\right)$ for $\mathrm{m} \in \mathbb{N}$ and $\mathrm{x} \geqslant 0$, then

$$
\alpha u_{\alpha, m+1}^{\rho}(x)=x\left[\left[u_{\alpha, m}^{\rho}(x)\right]^{\prime}+\frac{m(1+\rho)}{\rho} u_{\alpha, m-1}^{\rho}(x)\right]+\frac{m}{\rho} u_{\alpha, m}^{\rho}(x) .
$$

Proof. Using $x s_{\alpha, k}^{\prime}(x)=(k-\alpha x) s_{\alpha, k}(x)$ and $\left(t \theta_{\alpha, k}^{\rho}(t)\right)^{\prime}=\rho(k-\alpha t) \theta_{\alpha, k}^{\rho}(t)$, we have

$$
\begin{aligned}
x\left[\mathrm{u}_{\alpha, \mathrm{m}}^{\rho}(x)\right]^{\prime}= & \sum_{k=1}^{\infty} x s_{\alpha, k}^{\prime}(x) \int_{0}^{\infty} \theta_{\alpha, k}^{\rho}(t)(t-x)^{m} d t+\alpha e^{-\alpha x}(-x)^{m+1}+m(-x)^{m} e^{-\alpha x} \\
& -m x \sum_{k=1}^{\infty} s_{\alpha, k}(x) \int_{0}^{\infty} \theta_{\alpha, k}^{\rho}(t)(t-x)^{m-1} d t \\
= & \sum_{k=1}^{\infty}(k-\alpha x) s_{\alpha, k}(x) \int_{0}^{\infty} \theta_{\alpha, k}^{\rho}(t)(t-x)^{m} d t+\alpha e^{-\alpha x}(-x)^{m+1}-m x U_{\alpha, m-1}^{\rho}(x) \\
= & \sum_{k=1}^{\infty} s_{\alpha, k}(x) \int_{0}^{\infty}[(k-\alpha t)+\alpha(t-x)] \theta_{\alpha, k}^{\rho}(t)(t-x)^{m} d t \\
& +\alpha e^{-\alpha x}(-x)^{m+1}-m x U_{\alpha, m-1}^{\rho}(x) \\
= & \frac{1}{\rho} \sum_{k=1}^{\infty} s_{\alpha, k}(x) \int_{0}^{\infty}\left[t \theta_{\alpha, k}^{\rho}(t)\right]^{\prime}(t-x)^{m} d t+\alpha U_{\alpha, m+1}^{\rho}(x)-m x U_{\alpha, m-1}^{\rho}(x) .
\end{aligned}
$$

Integrating by parts the last integral, we have

$$
\begin{aligned}
x\left[\mathrm{U}_{\alpha, m}^{\rho}(x)\right]^{\prime}= & -\frac{\mathrm{m}}{\rho} \sum_{k=1}^{\infty} s_{\alpha, k}(x) \int_{0}^{\infty} t \theta_{\alpha, k}^{\rho}(t)(t-x)^{m-1} d t+\alpha U_{\alpha, m+1}^{\rho}(x)-m x u_{\alpha, m-1}^{\rho}(x) \\
= & -\frac{m}{\rho}\left[u_{\alpha, m}^{\rho}(x)-(-x)^{m} e^{-\alpha x}\right] \\
& +-\frac{m}{\rho}\left[x u_{\alpha, m-1}^{\rho}(x)-x(-x)^{m-1} e^{-\alpha x}\right]-m x u_{\alpha, m-1}^{\rho}(x)+\alpha u_{\alpha, m+1}^{\rho}(x) .
\end{aligned}
$$

Thus, we get

$$
\alpha u_{\alpha, m+1}^{\rho}(x)=x\left[\left[u_{\alpha, m}^{\rho}(x)\right]^{\prime}+\frac{m(1+\rho)}{\rho} u_{\alpha, m-1}^{\rho}(x)\right]+\frac{m}{\rho} u_{\alpha, m}^{\rho}(x) .
$$

Remark 2.5. From Lemma 2.4, it can easily be seen that

$$
u_{\alpha, 0}^{\rho}(x)=1, \quad u_{\alpha, 1}^{\rho}(x)=0, \quad u_{\alpha, 2}^{\rho}(x)=\frac{(\rho+1) x}{\alpha \rho}, u_{\alpha, 3}^{\rho}(x)=\frac{(\rho+1)(\rho+2) x}{\alpha^{2} \rho^{2}},
$$


and

$$
\mathrm{u}_{\alpha, 4}^{\rho}(x)=\frac{3(\rho+1)^{2} \chi^{2}}{\alpha^{2} \rho^{2}}+\frac{(\rho+1)(\rho+2)(\rho+3) x}{\alpha^{3} \rho^{3}} .
$$

Lemma 2.6. There exist the polynomials $\mathrm{q}_{\mathrm{i}, \mathrm{j}, \mathrm{r}}(\mathrm{x})$ independent of $\alpha$ and $\mathrm{k}$ such that

$$
x^{r} \frac{d^{r}}{d x^{r}} s_{\alpha, k}(x)=\sum_{\substack{2 i+j \leqslant r \\ i, j \geqslant 0}} \alpha^{i}(k-\alpha x)^{j} q_{i, j, r}(x) s_{\alpha, k}(x) .
$$

\section{Simultaneous approximation}

In this section we establish a Voronovskaja type asymptotic formula and error estimation in simultaneous approximation.

For $\gamma>0$, we denote the class of functions as

$$
\mathrm{C}_{\gamma}[0, \infty)=\left\{f \in \mathrm{C}[0, \infty):|\mathrm{f}(\mathrm{t})| \leqslant M t^{\gamma}, M>0\right\} .
$$

The norm $-\|\cdot\|_{\gamma}$ on this class of functions is defined as

$$
\|f\|_{\gamma}=\sup _{x \in[0, \infty)}|f(t)| t^{-\gamma}
$$

Theorem 3.1. Let $\mathrm{f} \in \mathrm{C}_{\gamma}[0, \infty)$. If $\mathrm{f}^{(\mathrm{r}+2)}$ exists at a point $\mathrm{x} \in(0, \infty)$, then for $\gamma>\mathrm{r}+2$, we have

$$
\lim _{\alpha \rightarrow \infty} \alpha\left(\left(\frac{d^{r}}{d w^{r}} L_{\alpha}^{\rho}(f, w)\right)_{w=x}-f^{(r)}(x)\right)=\frac{(\rho+1) r}{2 \rho} f^{(r+1)}(x)+\frac{(\rho+1) x}{2 \rho} f^{(r+2)}(x) .
$$

Further, if $\mathrm{f}^{(\mathrm{r}+2)}$ is continuous on $(\mathrm{a}-\eta, \mathrm{b}+\eta) \subset(0, \infty), \eta>0$, then the limit in (3.1) holds uniformly in $[\mathrm{a}, \mathrm{b}]$. Proof. From the Taylor's theorem, we may write

$$
f(t)=\sum_{v=0}^{r+2} \frac{f^{(v)}(x)}{v !}(t-x)^{v}+\psi(t, x)(t-x)^{r+2}, t \in[0, \infty),
$$

where the function $\psi(t, x) \rightarrow 0$ as $t \rightarrow x$. From equation (3.2), we obtain

$$
\begin{aligned}
\left(\frac{\mathrm{d}^{r}}{\mathrm{~d} w^{\mathrm{r}}} \mathrm{L}_{\alpha}^{\rho}(\mathrm{f}(\mathrm{t}), w)\right)_{w=x}= & \sum_{v=0}^{r+2} \frac{\mathrm{f}^{(v)}(\mathrm{x})}{v !}\left(\frac{\mathrm{d}^{\mathrm{r}}}{\mathrm{d} w^{\mathrm{r}}} \mathrm{L}_{\alpha}^{\rho}\left((\mathrm{t}-\mathrm{x})^{v}, w\right)\right)_{w=x} \\
& +\left(\frac{\mathrm{d}^{\mathrm{r}}}{\mathrm{d} w^{\mathrm{r}}} \mathrm{L}_{\alpha}^{\rho}\left(\psi(\mathrm{t}, x)(\mathrm{t}-x)^{\mathrm{r}+2}, w\right)\right)_{w=x} \\
= & \sum_{v=0}^{r+2} \frac{\mathrm{f}^{(v)}(\mathrm{x})}{v !} \sum_{j=0}^{v}\left(\begin{array}{l}
v \\
j
\end{array}\right)(-x)^{v-j}\left(\frac{\mathrm{d}^{\mathrm{r}}}{\mathrm{d} w^{\mathrm{r}}} \mathrm{L}_{\alpha}^{\rho}\left(\mathrm{t}^{j}, w\right)\right)_{w=x} \\
& +\left(\frac{\mathrm{d}^{\mathrm{r}}}{\mathrm{d} w^{\mathrm{r}}} \mathrm{L}_{\alpha}^{\rho}\left(\psi(\mathrm{t}, \mathrm{x})(\mathrm{t}-\mathrm{x})^{\mathrm{r}+2}, w\right)\right)_{w=x} \\
:= & \mathrm{I}_{1}+\mathrm{I}_{2}, \quad \text { (say). }
\end{aligned}
$$

Now, we estimate $I_{1}$.

$$
\mathrm{I}_{1}=\sum_{v=0}^{r-1} \frac{\mathrm{f}^{(v)}(\mathrm{x})}{v !} \sum_{j=0}^{v}\left(\begin{array}{l}
v \\
j
\end{array}\right)(-x)^{v-\mathrm{j}}\left(\frac{\mathrm{d}^{\mathrm{r}}}{\mathrm{d} w^{\mathrm{r}}} \mathrm{L}_{\alpha}^{\rho}\left(\mathrm{t}^{\mathrm{j}}, w\right)\right)_{w=x}
$$




$$
\begin{aligned}
& +\frac{f^{(r)}(x)}{r !} \sum_{j=0}^{r}\left(\begin{array}{l}
r \\
j
\end{array}\right)(-x)^{r-j}\left(\frac{d^{r}}{d w^{r}} L_{\alpha}^{\rho}\left(t^{j}, w\right)\right)_{w=x} \\
& +\frac{f^{(r+1)}(x)}{(r+1) !} \sum_{j=0}^{r+1}\left(\begin{array}{c}
r+1 \\
j
\end{array}\right)(-x)^{r+1-j}\left(\frac{d^{r}}{d w^{r}} L_{\alpha}^{\rho}\left(t^{j}, w\right)\right)_{w=x} \\
& +\frac{f^{(r+2)}(x)}{(r+2) !} \sum_{j=0}^{r+2}\left(\begin{array}{c}
r+2 \\
j
\end{array}\right)(-x)^{r+2-j}\left(\frac{d^{r}}{d w^{r}} L_{\alpha}^{\rho}\left(t^{j}, w\right)\right)_{w=x} \\
& =\frac{f^{(r)}(x)}{r !}\left(\frac{d^{r}}{d w^{r}} L_{\alpha}^{\rho}\left(t^{r}, w\right)\right)_{w=x} \\
& +\frac{f^{(r+1)}(x)}{(r+1) !}\left[(r+1)(-x)\left(\frac{d^{r}}{d w^{r}} L_{\alpha}^{\rho}\left(t^{r}, w\right)\right)_{w=x}+\left(\frac{d^{r}}{d w^{r}} L_{\alpha}^{\rho}\left(t^{r+1}, w\right)\right)_{w=x}\right] \\
& +\frac{f^{(r+2)}(x)}{(r+2) !}\left[\frac{(r+2)(r+1)}{2} x^{2}\left(\frac{d^{r}}{d w^{r}} L_{\alpha}^{\rho}\left(t^{r}, w\right)\right)_{w=x}\right. \\
& \left.+(r+2)(-x)\left(\frac{d^{r}}{d w^{r}} L_{\alpha}^{\rho}\left(t^{r+1}, w\right)\right)_{w=x}+\left(\frac{d^{r}}{d w^{r}} L_{\alpha}^{\rho}\left(t^{r+2}, w\right)\right)_{w=x}\right] .
\end{aligned}
$$

Applying Lemma 2.2, we have

$$
\begin{aligned}
I_{1}= & \frac{f^{(r)}(x)}{r !} r !+\frac{f^{(r+1)}(x)}{(r+1) !}\left[(r+1)(-x) \cdot r !+(r+1) ! x+\frac{\rho+1}{2 \alpha \rho}(r+1) r . r !\right] \\
& +\frac{f^{(r+2)}(x)}{(r+2) !}\left[\frac{(r+2)(r+1)}{2} \chi^{2} \cdot r !+(r+2)(-x)\left((r+1) ! x+\frac{\rho+1}{2 \alpha \rho}(r+1) r . r !\right)\right. \\
& +\frac{(r+2) !}{2} \chi^{2}+(r+1)(r+2) \frac{(\rho+1)}{2 \alpha \rho}(r+1) ! x \\
& \left.+\frac{(\rho+1)}{24(\alpha \rho)^{2}} \cdot(r+2)(r+1) r[(3 r+1) \rho+3 r+5] r !\right]
\end{aligned}
$$

Thus

$$
\lim _{\alpha \rightarrow \infty} \alpha\left(\left(\frac{d^{r}}{d w^{r}} L_{\alpha}^{\rho}(f, w)\right)_{w=x}-f^{(r)}(x)\right)=\left[f^{(r+1)}(x) \frac{(\rho+1) r}{2 \rho}+f^{(r+2)}(x) \frac{(\rho+1) x}{2 \rho}+\lim _{\alpha \rightarrow \infty} \alpha I_{2}\right] .
$$

In order to complete the proof, it is sufficient to show that $\lim _{\alpha \rightarrow \infty} \alpha \mathrm{I}_{2} \rightarrow 0$. We proceed as follows.

Next, in view of Lemma 2.6, we have

$$
\begin{aligned}
\left|I_{2}\right| \leqslant & \sum_{k=1}^{\infty} \sum_{\substack{i+j, j \leqslant r \\
i, j \geqslant 0}} \alpha^{i}|k-\alpha x|^{j} \frac{\left|q_{i, j, r}(x)\right|}{x^{r}} s_{\alpha, k}(x) \int_{0}^{\infty} \theta_{\alpha, k}^{\rho}(t)|\psi(t, x)| .|t-x|^{r+2} d t \\
& +\left|\psi(0, x)(-x)^{r+2}\right|\left(\frac{d^{r}}{d \omega^{r}} s_{\alpha, 0}(\omega)\right)_{\omega=x}=\Delta_{1}+\Delta_{2} .
\end{aligned}
$$

Now, we estimate $\Delta_{1}$. Since $\psi(t, x) \rightarrow 0$ as $t \rightarrow x$, for a given $\epsilon>0$ there exists a $\delta>0$ such that $|\psi(t, x)|<\epsilon$ whenever $|t-x|<\delta$. For $|t-x| \geqslant \delta$, we have $\left|(t-x)^{r+2} \psi(t, x)\right| \leqslant M|t-x|^{\gamma}$, for some $M>0$. Thus, from equation (3.3) we may write

$$
\begin{aligned}
\left|\Delta_{1}\right| \leqslant & \sum_{k=1}^{\infty} \sum_{\substack{2 i+j \leqslant r \\
i, j \geqslant 0}} \alpha^{i}|k-\alpha x|^{j} \frac{\left|q_{i, j, r}(x)\right|}{x^{r}} s_{\alpha, k}(x)\left(\epsilon \int_{|t-x|<\delta} \theta_{\alpha, k}^{\rho}(t)|t-x|^{r+2} d t\right. \\
& \left.+M \int_{|t-x| \geqslant \delta} \theta_{\alpha, k}^{\rho}(t)|t-x|^{\gamma} d t\right):=J_{1}+J_{2} .
\end{aligned}
$$


Let $K=\sup _{\substack{2 i+j \leqslant r \\ i, j \geqslant 0}} \frac{\left|q_{i, j, r}(x)\right|}{x^{r}}$. Using Schwarz inequality, Lemma 2.1, and Lemma 2.2, we have

$$
\begin{aligned}
J_{1} & =\epsilon K \sum_{\substack { k=1 \\
\begin{subarray}{c}{2 i+j \leqslant r \\
i, j \geqslant 0{ k = 1 \\
\begin{subarray} { c } { 2 i + j \leqslant r \\
i , j \geqslant 0 } }\end{subarray}}^{\infty} \alpha^{i}|k-\alpha x|^{j} s_{\alpha, k}(x)\left(\int_{0}^{\infty} \theta_{\alpha, k}^{\rho}(t) d t\right)^{1 / 2}\left(\int_{0}^{\infty} \theta_{\alpha, k}^{\rho}(t)|t-x|^{2 r+4} d t\right)^{1 / 2} \\
& \leqslant \epsilon K \sum_{\substack{2 i+j \leqslant r \\
i, j \geqslant 0}} \alpha^{i+j}\left(\sum_{k=0}^{\infty} s_{\alpha, k}(x)\left(\frac{k}{\alpha}-x\right)^{2 j}-x^{2 j} s_{\alpha, 0}(x)\right)^{1 / 2}\left(L_{\alpha}^{\rho}\left((t-x)^{2 r+4}, x\right)-x^{2 r+4} s_{\alpha, 0}(x)\right)^{1 / 2} \\
& =\epsilon \sum_{\substack{2 i+j \leqslant r \\
i, j \geqslant 0}} \alpha^{i+j}\left\{O\left(\frac{1}{\alpha^{j}}\right)+O\left(\frac{1}{\alpha^{s}}\right)\right\}^{1 / 2}\left\{O\left(\frac{1}{\alpha^{r+2}}\right)+O\left(\frac{1}{\alpha^{p}}\right)\right\}^{1 / 2} \text { for any } s, p>0 .
\end{aligned}
$$

Choose $s$ and $p$ such that $s>j$ and $p>r+2$

$$
J_{1} \leqslant \epsilon \sum_{\substack{2 i+j \leqslant r \\ i, j \geqslant 0}} \alpha^{i+j} O\left(\frac{1}{\alpha^{j / 2}}\right) O\left(\frac{1}{\alpha^{r / 2+1}}\right)=\epsilon \cdot O\left(\alpha^{-1}\right) .
$$

Since $\epsilon>0$ is arbitrary, $\alpha \mathrm{J}_{1} \rightarrow 0$ as $\alpha \rightarrow \infty$.

Again, using Schwarz inequality, Lemma 2.1, and Lemma 2.4, we obtain

$$
\begin{aligned}
J_{2} \leqslant & M_{1} \sum_{\substack{2 i+j \leqslant r \\
i, j \geqslant 0}} \alpha^{i+j}\left(\sum_{k=0}^{\infty}\left(\frac{k}{\alpha}-x\right)^{2 j} s_{\alpha, k}(x)-x^{2 j} s_{\alpha, 0}(x)\right)^{1 / 2} \\
& \times\left(\sum_{\substack{k=1 \\
\infty}}^{\infty} s_{\alpha, k}(x) \int_{|t-x| \geqslant \delta} \theta_{\alpha, k}^{\rho}(t)(t-x)^{2 \gamma} d t\right)^{1 / 2} \\
\leqslant & M_{1} \sum_{\substack{2 i+j \leqslant r \\
i, j \geqslant 0}} \alpha^{i+j}\left\{O\left(\frac{1}{\alpha^{j}}\right)+O\left(\frac{1}{\alpha^{p}}\right)\right\}^{1 / 2}\left\{O\left(\frac{1}{\alpha^{\gamma}}\right)\right\}^{1 / 2} \text { for any } p>0
\end{aligned}
$$

Choose $p$ such that $p>j$

$$
J_{2} \leqslant M_{1} \sum_{\substack{2 i+j \leqslant r \\ i, j \neq 0}} \alpha^{i+j} O\left(\frac{1}{\alpha^{j / 2}}\right) O\left(\frac{1}{\alpha^{\gamma / 2}}\right)=M_{1} O\left(\frac{1}{\alpha^{(\gamma-r) / 2}}\right),
$$

which implies that $\alpha J_{2} \rightarrow 0$, as $\alpha \rightarrow \infty$ on choosing $\gamma>r+2$.

From the above estimates of $J_{1}$ and $J_{2}, \alpha \Delta_{1} \rightarrow 0$, as $\alpha \rightarrow \infty$.

Next, we estimate $\Delta_{2}$.

$$
\left|\Delta_{2}\right|=\left|\psi(0, x)(-x)^{r}\right|\left(\frac{d^{r}}{d \omega^{r}} s_{\alpha, 0}(\omega)\right)_{\omega=x}
$$

Since $\left|\psi(0, x)(-x)^{r}\right|<N_{1}$ for some $N_{1}>0$, we get $\left(\frac{d^{r}}{d \omega^{r}} s_{n, 0}(\omega)\right)_{\omega=x}=\left[\frac{d^{r}}{d \omega^{r}}\left(e^{-\alpha w}\right)\right]_{\omega=x} \rightarrow 0$ as $\alpha \rightarrow \infty$, which yields that $\Delta_{2} \rightarrow 0$ as $\alpha \rightarrow \infty$. By combining the estimates of $\Delta_{1}$ and $\Delta_{2}$, we obtain $\alpha \mathrm{I}_{2} \rightarrow 0$ as $\alpha \rightarrow \infty$. Thus, from the estimates of $I_{1}$ and $I_{2}$, the required result follows.

To prove the uniformity assertion, it is sufficient to remark that $\delta(\epsilon)$ in the above proof can be chosen to be independent of $x \in[a, b]$ and also that the other estimates hold uniformity in $x \in[a, b]$. This completes the proof. Combining the estimates of $\mathrm{I}_{1}$ and $\mathrm{I}_{2}$, we get the required result. 
Theorem 3.2. Let $\mathrm{f} \in \mathrm{C}_{\gamma}[0, \infty)$ for some $\gamma>0$ and $\mathrm{r} \leqslant \mathrm{q} \leqslant \mathrm{r}+2$. If $\mathrm{f}^{(\mathrm{q})}$ exists and is continuous on $(a-\eta, b+\eta) \subset(0, \infty), \eta>0$, then for $\alpha$ sufficiently large, we have

$$
\left\|\left(L_{\mathfrak{n}}^{\rho}(f, .)\right)^{(r)}-f^{(r)}\right\| \leqslant \frac{C_{1}}{\alpha}\left(\sum_{j=r}^{q}\left\|f^{(j)}\right\|\right)+\frac{C_{2}}{\sqrt{\alpha}} \omega\left(f^{(r+1)}, \alpha^{-1 / 2}\right)+O\left(\alpha^{-2}\right),
$$

where $C_{1}$ and $C_{2}$ are absolute constants independent of $f$ and $\alpha$, norm is sup-norm on $[a, b]$ and $\omega(f, \delta)$ is the modulus of continuity of $f$ on the interval $(a-\eta, b+\eta)$.

Proof. Using Taylor's finite expansion of $f$, we can write

$$
f(t)=\sum_{j=0}^{q} \frac{f^{(j)}(x)}{j !}(t-x)^{j}+\frac{f^{(q)}(\xi)-f^{(q)}(x)}{q !}(t-x)^{q} \chi(t)+h(t, x)(1-\chi(t))
$$

where $\xi$ lies between $t$ and $\chi$ and $\chi(t)$ is the characteristic function of $(a-\eta, b+\eta)$. For $t \in(a-\eta, b+\eta)$ and $x \in[a, b]$, we have

$$
f(t)=\sum_{j=0}^{q} \frac{f^{(j)}(x)}{j !}(t-x)^{j}+\frac{f^{(q)}(\xi)-f^{(q)}(x)}{q !}(t-\xi)^{q}
$$

For $t \in(0, \infty) \backslash(a-\eta, b+\eta)$ and $x \in[a, b]$, we set

$$
h(t, x)=f(t)-\sum_{j=0}^{q} \frac{f^{(j)}(x)}{j !}(t-x)^{j}
$$

We have

$$
\begin{aligned}
\left(L_{\mathfrak{n}}^{\rho}(f, x)\right)^{(r)}-f^{(r)}(x)= & {\left[\sum_{j=0}^{q} \frac{f^{(j)}(x)}{j !} \int_{0}^{\infty}\left[k_{\alpha}^{\rho}(x, t)\right]^{(r)}(t-x)^{j} d t-f^{(r)}(x)\right] } \\
& +\int_{0}^{\infty}\left[k_{\alpha}^{\rho}(x, t)\right]^{(r)} \frac{f^{(q)}(\xi)-f^{(q)}(x)}{q !}(t-x)^{q} \chi(t) d t \\
& +\int_{0}^{\infty}\left[k_{\alpha}^{\rho}(x, t)\right]^{(r)} h(t, x)(1-\chi(t)) d t \\
:= & I_{1}+I_{2}+I_{3} .
\end{aligned}
$$

Using Lemma 2.2, we have

$$
\begin{aligned}
I_{1}= & \sum_{j=0}^{q} \frac{f^{(j)}(x)}{j !} \sum_{i=0}^{j}\left(\begin{array}{l}
j \\
i
\end{array}\right)(-x)^{j-i} \frac{d^{r}}{d x^{r}} \int_{0}^{\infty}\left[k_{\alpha}^{\rho}(x, t)\right]^{(r)} t^{i} d t-f^{(r)}(x) \\
= & \sum_{j=0}^{q} \frac{f^{(j)}(x)}{j !} \sum_{i=0}^{j}\left(\begin{array}{l}
j \\
i
\end{array}\right)(-x)^{j-i} \frac{d^{r}}{d x^{r}}\left[x^{i}+\frac{(\rho+1)}{2 \alpha \rho} \cdot i(i-1) x^{i-1}\right. \\
& \left.+\frac{(\rho+1)}{24(\alpha \rho)^{2}} \cdot i(i-1)(i-2)[(3 i-5) \rho+3 i-1] x^{i-2}+\cdots\right]-f^{(r)}(x) .
\end{aligned}
$$

Hence

$$
\left\|\mathrm{I}_{1}\right\| \leqslant \frac{\mathrm{C}_{1}}{\alpha}\left(\sum_{j=0}^{\mathrm{q}}\left\|\mathrm{f}^{(\mathrm{j})}\right\|\right)+\mathrm{O}\left(\alpha^{-2}\right)
$$


Next, for $\delta>0$, we have

$$
\begin{aligned}
& I_{2}=\int_{0}^{\infty}\left|\left[k_{\alpha}^{\rho}(x, t)\right](r)\right| \frac{\left|f^{(q)}(\xi)-f^{(q)}(x)\right|}{q !}|t-x|^{q} \chi(t) d t \\
& \leqslant \frac{\omega\left(f^{(q)}, \delta\right)}{q !} \int_{0}^{\infty}\left|\left[k_{\alpha}^{\rho}(x, t)\right]^{(r)}\right|\left(1+\frac{|t-x|}{\delta}\right)|t-x|^{q} d t \\
& \leqslant \frac{\omega\left(f^{(q)}, \delta\right)}{q !}\left[\sum_{k=1}^{\infty}\left|s_{\alpha, k}^{(r)}(x)\right| \int_{0}^{\infty} \theta_{\alpha, k}^{\rho}(t)\left(|t-x|^{q}+\delta^{-1}|t-x|^{q+1}\right) d t+\alpha^{r} e^{-\alpha x}\left(|x|^{q}+\delta^{-1}|x|^{q+1}\right)\right] \text {. }
\end{aligned}
$$

To evaluate $I_{2}$, we consider the following and applying Lemmas 2.6, 2.1, 2.4 in the next steps,

$$
\begin{aligned}
& \sum_{k=1}^{\infty}\left|s_{\alpha, k}^{(r)}(x)\right| \int_{0}^{\infty} \theta_{\alpha, k}^{\rho}(t)|t-x|^{m} d t \\
& \quad \leqslant \sum_{k=1}^{\infty} \sum_{\substack{2 i+j \leqslant r \\
i, j \geqslant 0}} \alpha^{i}|k-\alpha x|^{j} \frac{q_{i, j, r}(x)}{x^{r}} s_{\alpha, k}(x) \int_{0}^{\infty} \theta_{\alpha, k}^{\rho}(t)|t-x|^{m} d t \\
& \quad \leqslant C \sum_{\substack{2 i+j \leqslant r \\
i, j \geqslant 0}} \alpha^{i} \sum_{k=1}^{\infty} s_{\alpha, k}(x)|k-\alpha x|^{j} \int_{0}^{\infty} \theta_{\alpha, k}^{\rho}(t)|t-x|^{m} d t \\
& \leqslant C \sum_{\substack{2 i+j \leqslant r \\
i, j \geqslant 0}} \alpha^{i} \sum_{k=1}^{\infty} s_{\alpha, k}(x)|k-\alpha x|^{j}\left[\left(\int_{0}^{\infty} \theta_{\alpha, k}^{\rho}(t) d t\right)^{1 / 2}\left(\int_{0}^{\infty} \theta_{\alpha, k}^{\rho}(t)(t-x)^{2 m} d t\right)^{1 / 2}\right] \\
& \leqslant C \sum_{\substack{2 i+j \leqslant r \\
i, j \geqslant 0}} \alpha^{i}\left(\sum_{k=1}^{\infty} s_{\alpha, k}(x)(k-\alpha x)^{2 j}\right)^{1 / 2}\left(\sum_{k=1}^{\infty} s_{\alpha, k}(x) \int_{0}^{\infty} \theta_{\alpha, k}^{\rho}(t)(t-x)^{2 m} d t\right)^{1 / 2} \\
& \quad=C \sum_{\substack{2 i+j \leqslant r \\
i, j \geqslant 0}} \alpha^{i} \cdot O\left(\alpha^{(j-m) / 2}\right)=O\left(\alpha^{(r-m) / 2}\right)
\end{aligned}
$$

uniformly in $x$, with $C=\sum_{\substack{2 i+j \leqslant r \\ i, j \geqslant 0}} \sup _{x \in[a, b]} \frac{\left|q_{i, j, r}(x)\right|}{x^{r}}$.

Thus choosing $\delta=\alpha^{-1 / 2}$ and using above we get for any $s>0$

$$
\left\|I_{2}\right\| \leqslant \frac{\omega\left(f^{(q)}, \alpha^{-1 / 2}\right)}{q !}\left[O\left(\alpha^{(r-q) / 2}\right)+\alpha^{1 / 2} O\left(\alpha^{(r-q-1) / 2}\right)+O\left(\alpha^{-s}\right)\right] \leqslant C_{2} \alpha^{-(q-r) / 2} \omega\left(f^{(q)}, \alpha^{-1 / 2}\right) .
$$

We choose a $\delta \in(0, \eta)$. Using Lemma 2.6, we obtain

$$
\left|I_{3}\right| \leqslant \sum_{k=1}^{\infty} \sum_{\substack{2+i j \leqslant r \\ i, j \geqslant 0}} \alpha^{i}|k-\alpha x|^{j} \frac{\left|q_{i, j, r}(x)\right|}{x^{r}} s_{\alpha, k}(x) \int_{|t-x| \geqslant \delta} \theta_{\alpha, k}^{\rho}(t)|h(t, x)| d t .
$$

If $\beta$ is any integer greater than or equal to $\{\gamma, q\}$ we can find a constant $K$ such that $|h(t, x)| \leqslant K|t-x|^{\beta}$ for $|t-x| \geqslant \delta$. Applying Schwarz inequality and Lemma 2.4, we get $I_{3}=\mathrm{O}\left(\alpha^{-m}\right)$ for any $m>0$ uniformly on $[a, b]$. Combining the estimates of $I_{1}, I_{2}, I_{3}$, we get the desired result.

For sufficiently small $\eta>0$, the Steklov mean $f_{\eta, 2}$ of 2 nd order corresponding to $f \in C_{\gamma}[0, \infty)$ and $t \in I_{i}=\left[a_{i}, b_{i}\right], i=1,2$ is defined as follows:

$$
f_{\eta, 2}(t)=\eta^{-2} \int_{-\eta / 2}^{\eta / 2} \int_{-\eta / 2}^{\eta / 2}\left(f(t)-\Delta_{h}^{2} f(t)\right) d t_{1} d t_{2},
$$


where $h=\frac{t_{1}+t_{2}}{2}$ and $\Delta_{h}^{2}$ is the second order forward difference operator with step length $h$. The following properties are satisfied (see $[7,11]$ and references therein):

(i) $f_{\eta, 2}$ has continuous derivatives up to order 2 over $I_{1}$;

(ii) $\left\|f_{\eta, 2}^{(r)}\right\|_{C\left(I_{2}\right)} \leqslant C \eta^{-r} \omega_{r}\left(f, \eta, I_{2}\right), r=1,2$;

(iii) $\left\|f-f_{\eta, 2}\right\|_{C\left(I_{2}\right)} \leqslant C \omega_{2}\left(f, \eta, I_{1}\right)$;

(iv) $\left\|f_{\eta, 2}\right\|{ }_{C\left(I_{2}\right)} \leqslant C\|f\|_{C\left(I_{1}\right)} \leqslant C\|f\|_{\gamma}$,

where $C$ is a constant not necessarily the same at each occurrence and is independent of $f$ and $\eta$.

Lemma 3.3 ([3]). Let $f \in C(I)$. Then,

$$
\left\|f_{\eta, 2 k}^{(i)}\right\|_{C(I)} \leqslant C_{i}\left\{\left\|f_{\eta, 2}\right\|_{C(I)}+\left\|f_{\eta, 2}^{(2 k)}\right\|_{C(I)}\right\}, i=1,2, \ldots, 2 k-1,
$$

where $C_{i}$ 's are certain constants independent of $f$.

Theorem 3.4. Let $\mathrm{f} \in \mathrm{C}_{\gamma}[0, \infty)$ for some $\gamma>0$ and $0<\mathrm{a}<\mathrm{a}_{1}<\mathrm{b}_{1}<\mathrm{b}<\infty$. Then for $\alpha$ sufficiently large, we have

$$
\left\|\left(L_{\alpha}^{\rho(r)}(f, .)\right)-f^{(r)}\right\|_{C\left(I_{1}\right)} \leqslant K_{1} \omega_{2}\left(f^{(r)}, \alpha^{-1 / 2}, I\right)+K_{2} \alpha^{-1}\|f\|_{\gamma}
$$

where $\mathrm{K}_{1}=\mathrm{K}_{1}(\mathrm{r})$ and $\mathrm{K}_{2}=\mathrm{K}_{2}(\mathrm{r}, \mathrm{f})$.

Proof. We can write

$$
\begin{aligned}
\left\|\left(L_{\alpha}^{\rho(r)}(f, .)\right)-f^{(r)}\right\|_{C\left(I_{1}\right)} & \leqslant\left\|L_{\alpha}^{\rho(r)}\left(\left(f-f_{\eta, 2}\right), .\right)\right\|_{C\left(I_{1}\right)}+\left\|\left(L_{\alpha}^{\rho(r)}\left(f_{\eta, 2}, .\right)\right)-f_{\eta, 2}^{(r)}\right\|_{C\left(I_{1}\right)}+\left\|f^{(r)}-f_{\eta, 2}^{(r)}\right\|_{C\left(I_{1}\right)} \\
& =M_{1}+M_{2}+M_{3} .
\end{aligned}
$$

Since $f_{\eta, 2}^{(r)}=\left(f^{(r)}\right)_{\eta, 2}$, hence by property (iii) of the Steklov mean, we get

$$
M_{3} \leqslant K_{1} \omega_{2}\left(f^{(r)}, \eta, I\right) .
$$

Next, applying Theorem 3.1 and Lemma 3.3, we obtain

$$
M_{2} \leqslant K_{2} \alpha^{-1} \sum_{i=r}^{r+2}\left\|f_{\eta, 2}^{(i)}\right\|_{C\left(I_{1}\right)} \leqslant K_{3} \alpha^{-1}\left\{\left\|f_{\eta, 2}\right\|_{C\left(I_{1}\right)}+\left\|f_{\eta, 2}^{(r+2)}\right\|_{C\left(I_{1}\right)}\right\} .
$$

By using properties (ii) and (iv) of Steklov mean, we get

$$
M_{2} \leqslant K_{4} \alpha^{-1}\left\{\|f\|_{\gamma}+\eta^{-2} \omega_{2}\left(f^{(r)}, \eta, I\right)\right\}
$$

Let $\mathrm{a}^{*}$ and $\mathrm{b}^{*}$ be such that $0<\mathrm{a}<\mathrm{a}^{*}<\mathrm{a}_{1}<\mathrm{b}_{1}<\mathrm{b}^{*}<\mathrm{b}<\infty$ and $\mathrm{I}^{*}$ denote the interval [a*, $\mathrm{b}^{*}$.

Now, we estimate $M_{1}$. Let $f-f_{\eta, 2} \equiv F$. By our hypothesis we can write

$$
F(t)=\sum_{m=0}^{r} \frac{F^{(m)}(x)}{m !}(t-x)^{m}+\frac{F^{(r)}(\xi)-F^{(r)}(x)}{r !}(t-x)^{r} \chi(t)+\theta(t, x)(1-\chi(t)),
$$

where $\xi$ lies between $t$ and $\chi$, and $\chi$ denotes the characteristic function of the interval $I^{*}$. For $t \in I^{*}$ and $x \in \mathrm{I}_{1}$, we get

$$
F(t)=\sum_{m=0}^{r} \frac{F^{(m)}(x)}{m !}(t-x)^{m}+\frac{F^{(r)}(\xi)-F^{(r)}(x)}{r !}(t-x)^{r}
$$


and for $\mathrm{t} \in[0, \infty) \backslash \mathrm{I}^{*}, x \in \mathrm{I}_{1}$ we set

$$
\theta(t, x)=F(t)-\sum_{m=0}^{r} \frac{F^{(m)}(x)}{m !}(t-x)^{m} .
$$

Operating $L_{\alpha}^{\rho(r)}$ on both sides of (3.4), we get three terms $J_{1}, J_{2}$, and $J_{3}$, corresponding to three terms in right hand side of (3.4). Using Theorem 3.1, we get

$$
\left|J_{1}\right| \leqslant\left\|f^{(r)}-f_{\eta, 2}^{(r)}\right\|_{C\left(I_{1}\right)} .
$$

Next, using Theorem 3.1, we obtain

$$
\left|J_{2}\right| \leqslant \frac{2\left\|F^{(r)}\right\|}{r !} L_{\alpha}^{\rho(r)}\left((t-x)^{r} \chi(t), x\right) \leqslant K_{5}\left\|f^{(r)}-f_{\eta, 2}^{(r)}\right\|_{C\left(I_{*}\right)}
$$

Lastly, we easily have

$$
\left|J_{3}\right|=L_{\alpha}^{\rho(r)}(1-\chi(t) \theta(t, x), x)=O\left(\alpha^{-s}\right) \text { for any } s>0 .
$$

Combining $\mathrm{J}_{1}-\mathrm{J}_{3}$, and from property (iii) of the Steklov mean, we obtain

$$
M_{1} \leqslant K_{6}\left\|f^{(r)}-f_{\eta, 2}^{(r)}\right\|_{C\left(I_{*}\right)} \leqslant K_{6} \omega_{2}\left(f^{(r)}, \eta, I\right) .
$$

Finally choosing $\eta=\alpha^{-1 / 2}$, the required result follows at once.

Remark 3.5. Proceeding along the lines of [9], one can extend the results for Păltănea type operators $L_{\alpha}^{\rho}(f, x)$. As the analysis is different, and due to the complicated form of these operators, it may be considered elsewhere.

\section{References}

[1] Z. Finta, V. Gupta, Direct and inverse estimates for Phillips type operators, J. Math. Anal. Appl., 303 (2005), 627-642. $1,2.1$

[2] S. G. Gal, V. Gupta, Complex form of a link operator between the Phillips and the Szász-Mirakjan operators, Results Math., 67 (2015), 381-393. 1

[3] S. Goldberg, A. Meir, Minimum moduli of ordinary differential operators, Proc. London Math. Soc., 23 (1971), 1-15. 3.3

[4] N. K. Govil, V. Gupta, M. A. Noor, Simultaneous approximation for the Phillips operators, Int. J. Math Math. Sci., 2006 (2006), 9 pages. 1

[5] V. Gupta, A note on modified Phillips operators, Southeast Asian Bull. Math., 34 (2010), 847-851. 1

[6] V. Gupta, On Approximation Properties of Szász-Mirakyan Operators, Springer Optim. Appl., New York, (2014). 1

[7] V. Gupta, R. P. Agarwal, Convergence Estimates in Approximation Theory, Springer, New York, (2014). 3

[8] V. Gupta, G. S. Srivastava, On the rate of convergence of Phillips operators for functions of bounded variation, Comment. Math., 36 (1996), 123-130. 1

[9] V. Gupta, G. Tachev, On approximation properties of Phillips operators preserving exponential functions, Mediterr. J. Math., 2017 (2017), 12 pages. 3.5

[10] M. Heilmann, G. Tachev, Commutativity, direct and strong converse results for Phillips operators, East J. Approx., 17 (2011), 299-317. 1

[11] E. Hewitt, K. Stromberg, Real and abstract Analysis, Springer-Verlag, New York, (1956). 3

[12] S. M. Mazhar, V. Totik, Approximation by modified Szasz operators, Acta Sci. Math., 49 (1985), 257-269. 1

[13] R. Pǎltănea, Modified Szász-Mirakjan operators of integral form, Carpathian J. Math., 24 (2008), 378-385. 1, 1

[14] R. Pǎltǎnea, Simultaneous approximation by a class of Szász Mirakjan operators, J. Appl. Funct. Anal., 9 (2014), 356-368. 2.2

[15] R. S. Phillips, An inversion formula for Laplace transforms and semi-groups of linear operators, Ann. of Math., 59 (1954), 325-356. 1

[16] O. Szasz, Generalization of S. Bernstein's polynomials to the infinite interval, J. Research Nat. Bur. Standards, 45 (1950), 239-245. 1

[17] G. Tachev, Approximation of bounded continuous functions by linear combinations of Phillips operators, Demonstr. Math., 47 (2014), 662-671. 1 